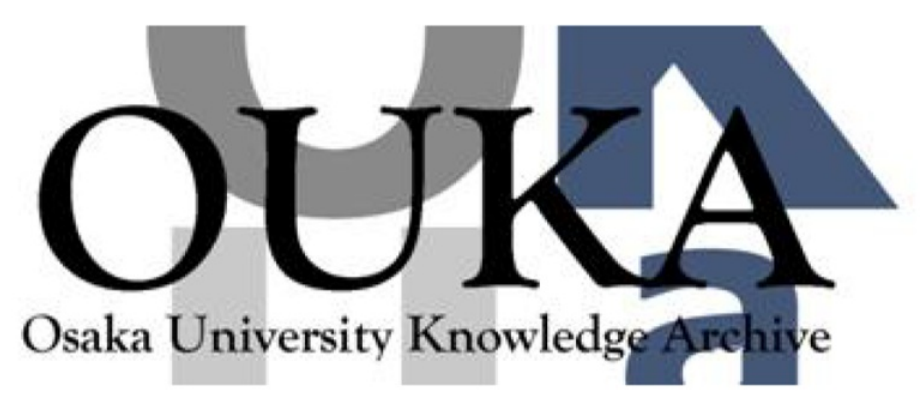

\begin{tabular}{|c|c|}
\hline Title & $\begin{array}{l}\text { Calibration-free portable Young' s-modulus } \\
\text { tester with isolated langasite oscillator }\end{array}$ \\
\hline Author (s) & Ogi, Hirotsugu; Sakamoto, Yuto; Hirao, Masahiko \\
\hline Citation & Ultrasonics. 54(7) p. 1963-p. 1966 \\
\hline Issue Date & $2014-05-15$ \\
\hline oaire:version & AM \\
\hline URL & https://hdl. handle. net/11094/84199 \\
\hline rights & $\begin{array}{l}\text { () } 2014 \text { Elsevier B.V. This manuscript version is } \\
\text { made avai lable under the Creative Commons } \\
\text { Attribution-NonCommercial-NoDerivatives } 4.0 \\
\text { International License. }\end{array}$ \\
\hline Note & \\
\hline
\end{tabular}

Osaka University Knowledge Archive : OUKA

https://ir. Library. osaka-u. ac. jp/

0saka University 


\section{Elsevier Editorial System(tm) for Ultrasonics \\ Manuscript Draft}

Manuscript Number:

Title: Calibration-free portable Young's-modulus tester with isolated langasite oscillator

Article Type: Research paper

Section/Category: Physical acoustics (A.G. Every)

Keywords: Young's modulus, ultrasonic resonator, electrodeless oscillator

Corresponding Author: Associate Professor Hirotsugu Ogi, Ph.D.

Corresponding Author's Institution: Osaka University

First Author: Hirotsugu Ogi, Ph.D.

Order of Authors: Hirotsugu Ogi, Ph.D.; Yuto Sakamoto; Masahiko Hirao

Abstract: A ballpoint-pen-type portable ultrasonic oscillator is developed for quantitative measurement of Young's modulus on a solid. It consists of an electrodeless rod-shaped langasite oscillator with a tungsten-carbide spherical-shaped tip at the end, permanent magnets for making a constant force at the contact interface, and antennas for exciting and detecting the longitudinal vibration contactlessly. The resonance frequency of the oscillator is changed by contact with the specimen, reflecting Young's modulus of the specimen at the contact area. The langasite oscillator is supported at the nodal points so that its acoustical contact occurs only at the specimen, making a calibration-free measurement realistic. Young's moduli of various specimens were evaluated within $15 \%$ error just by touching the specimens with the probe. The error becomes smaller than $10 \%$ for lower Young-modulus materials ( $150 \mathrm{GPa})$. 


\title{
Calibration-free portable Young's-modulus tester with isolated langasite oscillator
}

\author{
Hirotsugu Ogi, Yuto Sakamoto, Masahiko Hirao \\ Graduate School of Engineering Science, Osaka University
}

\begin{abstract}
A ballpoint-pen-type portable ultrasonic oscillator is developed for quantitative measurement of Young's modulus on a solid. It consists of an electrodeless rod-shaped langasite oscillator with a tungsten-carbide spherical-shaped tip at the end, permanent magnets for making a constant force at the contact interface, and antennas for exciting and detecting the longitudinal vibration contactlessly. The resonance frequency of the oscillator is changed by contact with the specimen, reflecting Young's modulus of the specimen at the contact area. The langasite oscillator is supported at the nodal points so that its acoustical contact occurs only at the specimen, making a calibration-free measurement realistic. Young's moduli of various specimens were evaluated within $15 \%$ error just by touching the specimens with the probe. The error becomes smaller than 10\% for lower Young-modulus materials $(<\sim 150 \mathrm{GPa})$.
\end{abstract}

Keywords: Young's modulus, ultrasonic resonator, electrodeless oscillator

\section{Introduction}

Young's modulus is the most important engineering elastic stiffness of a solid, and it is indispensable for designing any structures. Various methods were, therefore, proposed to measure Young's modulus. The tensile test and bending test are classical methods for measuring Young's modulus, where relationships between applied force and deformations are measured to deduce Young's modulus. These classical methods require specimens to be machined into specific shapes and they are inapplicable to small-size solids.

Email address: ogi@me.es.osaka-u.ac.jp (Hirotsugu Ogi) 
The acoustic methods, such as bar-resonance methods [1], pulse-echo methods [2], and resonant ultrasound spectroscopy [3, 4, 5], allow more accurate determination of the elastic constants with small specimens. (Comparison of these methods are discussed by Ledbetter et al. in detail [6].) However, they again need well-shaped specimens and high-skilled techniques. Indentation tests allow estimation of Young's modulus on solids, but they involve many ambiguous parameters, such as shape of indenter, elasitc-plastic behavior of the material, and so on $[7,8]$. Furthermore, all the previous methods require measurements of at least two quantities for deducing Young's modulus: Force and deformation in the tensile, bending and indentation tests, resonant frequency and dimension in the acoustic resonance methods, ultrasonic round-trip time and thickness in the pulse-echo method. These requirements have prevented us from making quick and easy evaluation of Young's modulus of arbitrarily-shaped solids.

In this study, we develop a portable instrument for quick evaluation of Young's modulus of a solid just by touching a probe to the solid surface. Only one quantity, the resonance frequency of the oscillator, yields Young's modulus without any other parameters. This method is based on resonant ultrasound microscopy (RUM) [9, 10, 11], which we developed for measuring local Young's modulus of solids. RUM allows one to measure Young's modulus images on a solid quantitatively, but it requires one unknown parameter, the radius of the diamond tip[11], and a large and expensive instrument for high-power excitation of the small electrodeless piezoelectric oscillator. The probe and instrument could never be mobile. Now, we adopt a larger oscillator with nearly perfect spherical-shaped tip at the end and install it into a ballpoint-pen like holder. Because of adopting higher-efficiency excitation mode, the instrument can be compact and mobile. Three key points for the calibration-free measurement have been achieved in this study: (i) acoustical isolation of the oscillator, (ii) use of perfect spherical-shaped tip, and (iii) constant biasing force.

\section{Measurement principle}

Resonance frequency of an oscillator increases when it contacts a solid, because elastic deformation occurs in the solid, raising the strain energy in the resonator system. The change in the resonance frequency then depends on the elastic stiffness of the solid in contact. It is then possible to determine the 
elastic constants of the solid through the change in the resonance frequency of the oscillator [11].

The contact interface between the tip and specimen can be considered as an elastic spring, which connects the oscillator and rigid surface as shown in Fig. 1, and the spring constant $K$ depends on elastic constants of the tip and specimen, the tip radius $R$, and the biasing force $F[11,12]$ as

$$
K=\sqrt[3]{6 R F E^{*}}
$$

Here $E^{*}$ denotes the effective contact Young's modulus defined by

$$
\frac{1}{E^{*}}=\frac{1-\nu_{t i p}^{2}}{E_{t i p}}+\frac{1-\nu_{s p e}^{2}}{E_{s p e}}
$$

Here, $E_{t i p}$ and $\nu_{t i p}$ are Young's modulus and Poisson's ratio of the tip, respectively, and $E_{\text {spe }}$ and $\nu_{\text {spe }}$ are those of specimen. When the oscillator shows a thin-rod shape (length $L$ and cross-section area $A$ ), its resonance frequency $f$ is given through a frequency equation [12]

$$
k L \tan (k L)=\frac{K}{K_{\text {osc }}}
$$

where $k=2 \pi f \sqrt{\rho / E_{\text {osc }}}$ denotes the wavenumber, and $K_{\text {osc }}=E_{\text {osc }} A / L$ is the equivalent spring constant of the oscillator. $E_{\text {osc }}$ is Young's modulus of the oscillator in the longitudinal direction. Figure 2 shows the relationship between the specimen's Young's modulus and the resonance frequency change calculated using Equations (1)-(3). As the tip radius increases, the sensitivity of the resonance frequency to specimen's Young's modulus increases, while the contact area becomes larger. The sensitivity deteriorates for a specimen with large Young's modulus, because the deformation in the tip contributes more to the resonance frequency rather than that in the specimen.

\section{Measurement system}

As shown above, the specimen's Young's modulus $E_{\text {spe }}$ is obtained by measuring the resonance frequency of the oscillator contacting the specimen in principle. However, for calibration-free measurement, three key points given in the introduction have to be accomplished. First, the acoustical contact to the oscillator has to be made only with the specimen, and any other contacts, even deposited electrodes and wires, must be eliminated because 
they also affect the resonance frequency. We used a square-column-shaped monocrystal langasite oscillator with $L=20 \mathrm{~mm}$ and $A=2.0 \times 2.0 \mathrm{~mm}^{2}$. The crystallographic [100] axis is directed along its length direction. The oscillator was supported by an O-ring at the four nodal points (apexes of squareshape cross-section at the center) of the fundamental rod vibrational mode as shown in Fig. 3 (a) to minimize the influence of contacts for support on the frequency response. We used antennas near the oscillator for exciting and detecting the resonance frequency of the electrodeless oscillator contactlessly [11]. The acoustical isolation of the langasite probe was achieved in these ways.

Second, the tip shape must be a perfect sphere for adopting Equations (1) and (2). We used a tungsten-carbide ball-bearing for this, whose sphericity is $0.64 \mu \mathrm{m}$ in diameter. (The diameter fluctuation is smaller than $0.016 \%$.) The ball-bearing was carefully polished using diamond slurries into a thin tip (0.3 $\mathrm{mm}$ height). The tip weight is much smaller than that of the oscillator (less than 2\%), affecting little the resonance frequency. It was bonded on the bottom surface of the langasite oscillator (Fig. 3(b)). We thus removed tip-shape ambiguity.

Third, we maintained a constant biasing force using the repulsive force of permanent magnets. As shown in Fig.3 (a) and Fig. 4, the holder, in which the O-ring and the permanent magnet are attached, smoothly slides along the outer guide together with the oscillator when the probe is pressed onto specimen, and it stops when the outer guide contacts the specimen surface. The distance between the two magnets is kept constant, giving a constant biasing force $(F=0.455 \mathrm{~N})$. This force is much larger than the weight of the holder involving the O-ring, magnet, and oscillator $(0.017 \mathrm{~N})$ and it is nearly constant even in case of inclined objects as shown in Fig. 4.

We chose the [100] direction of langasite to be the longitudinal direction for three reasons. First, Young's modulus in this direction is relatively smaller, and the sensitivity of the resonance frequency to the contact stiffness increases (see Equation (3)). Second, the absolute value of the piezoelectric coefficient $e_{11}$ is larger, allowing effective excitation and detection of the longitudinal vibration with the antenna configuration in Fig. 3(a), where the electric-field component in the [100] direction principally appears. Third, the temperature dependence of the resonance frequency is smaller. We measured it between 24 and $36{ }^{\circ} \mathrm{C}$ and obtained $\left(\frac{\partial f}{\partial T}\right) / f=2.4 \mathrm{ppm} / \mathrm{K}$. This value is significantly smaller than the change in the resonance frequency caused by contact with a specimen (> $3000 \mathrm{ppm})$. 
We excited the langasite oscillator by applying tone bursts with amplitude of $20 \mathrm{~V}_{p p}$ and duration of $10 \mathrm{~ms}$ to the generating antenna. After the excitation, the reverberating signals were detected by the receiving antenna. After amplified by $32 \mathrm{~dB}$, the waveform was digitally acquired with a portable digitizer, and Fourier transformation was performed to obtained the amplitude at the exciting frequency. By sweeping the frequency of the tone bursts and measuring the amplitude, we obtain the resonance spectrum. The Lorentzian-function fitting procedure determined the resonance frequency.

\section{Results and discussion}

Figure 5 shows resonance spectra measured in contact with various materials, clearly displaying that the resonance frequency increases as the specimen stiffens. At the noncontacting state, the resonance frequency is near 106 $\mathrm{kHz}$, while the theoretical value is estimated to be $118 \mathrm{kHz}$ using the reported elastic constants and mass density[13]. The peak amplitude decreases with the increase in Young's modulus of specimen. This is attributed to leakage of vibrational energy of the oscillator into the specimen because the acoustic impedance of the specimen approaches that of the tungsten-carbide tip.

We used literature values for the elastic properties of langasite[13] and tungsten carbide $\left(E_{t i p}=630 \mathrm{GPa}\right.$ and $\left.\nu_{t i p}=0.21\right)$. For the specimen's Poisson's ratio, we assumed $\nu_{\text {spe }}=0.25$, which, however, affects insignificantly the resultant Young's modulus (see Equation (2)). We can, therefore, determine the specimen's Young's modulus $E_{s p e}$ just by measuring the resonance frequency at the contact condition without using any other parameters. Figure 6 compares Young's moduli thus measured with reported values for various materials. Despite the calibration-free measurement, they agree with each other within $10 \%$ for materials with Young's modulus lower than $150 \mathrm{GPa}$. The difference, however, increased up to $15 \%$ for larger-Young-modulus materials, because the probe sensitivity deteriorates with the specimen's Young's modulus, as shown in Fig. 2. Other methods, such as the pulse-echo method, will provide more accurate stiffness, but they require well-shaped specimens, large and expensive instruments, and a long period of time for surface preparation. On the other hand, the present method allows a quick and pointwise measurement even for specimens with arbitrary configurations.

Error bars in Fig. 6 indicate standard deviation for five independent measurements on different points. The larger error bars for polycrystalline materials $(\mathrm{Cu}$, carbon steel, and $\mathrm{Ni})$ are caused by different effective Young's mod- 
ulus depending on the crystallographic orientation. For example, Young's moduli of $\mathrm{Cu}$ along [100], [110], and [111] are 65, 129, and $189 \mathrm{GPa}$, respectively. (The aggregated value is 126 GPa.) Therefore, measured Young's modulus is affected by orientation of grains involved in the detectable region in such a locally anisotropic material. Using the Willis approach for Hertzian contact with an anisotropic specimen[11, 14], we calculated the distribution of strain energy in the transverse-isotropically oriented (111) $\mathrm{Cu}$ specimen. The result is shown in Fig. 7. The contact radius $a$ is $17.7 \mu \mathrm{m}$ in this case, and the strain-energy penetration depth is close to the contact radius. Our probe is then sensitive to a volume of $\sim\left(\pi a^{2} \times a\right)$ at the surface region, and the measurement is affected by any stiffness variation with this volume order. It is possible to enlarge the mearuing volume either by increasing the biasing force or by using a larger radius tip.

\section{Conclusions}

We have successfully developed a portable Young's modulus tester using a langasite oscillator and a spherical-shaped tungsten-carbide tip. It easily and quickly measures Young's moduli of solids just by pressing the probe against the surface. Machining and preparation of specimen is basically unnecessary; only a $\sim 10$-mm-diameter flat surface is needed. The measurement accuracy is $10 \%$ for materials with Young's modulus lower than $150 \mathrm{GPa}$ without using any calibration procedures, although the error increased up to $15 \%$ for higher-modulus materials. Because of the portability, this system could be adopted for health monitoring and nondestructive evaluation of structures because local defects significantly lower the apparent elastic stiffness. 


\section{References}

[1] D.T. Read, H.M. Ledbetter, Elastic properties of a boron-aluminum composite at low temperatures, J. Appl. Phys. 48 (1977) 2827-2831.

[2] H.M. Ledbetter, Orthotropic elastic stiffnesses of a boron-aluminum composite, J. Appl. Phys. 50 (1979) 8247-8248.

[3] I. Ohno, Free vibration of a rectangular parallelepiped crystal and its application to determination of elastic constants of orthorhombic crystals, J. Phys. Earth 24 (1976) 355-379.

[4] A. Migliori, J.L. Sarrao, William M. Visscher, T.M. Bell, Ming Lei, Z. Fisk, R.G. Leisure, Resonant ultrasound spectroscopic techniques for measurement of the elastic moduli of solids, Physica B 183 (1993) 1-24.

[5] H. Ogi, K. Sato, T. Asada, M. Hirao, Complete mode identification for resonance ultrasound spectroscopy, J. Acoust. Soc. Am. 112 (2002) 2553-2557.

[6] H. Ledbetter, C. Fortunko, P. Heyliger, Orthotropic elastic constants of a boron-aluminum fiber-reinforced composite: An acoustic-resonancespectroscopy study, J. Appl. Phys. 78 (1995) 1542-1546.

[7] W.C. Oliver, G.M. Pharr, An improved technique for determining hardness and elastic modulus using load and displacement sensing indentation experiments, J. Mater. Res. 6 (1992) 1564-1583.

[8] J.M. Antunes, J.V. Fernandes, N.A. Sakharova, M.C. Oliveira, L.F. Menezes, On the determination of the Youngs modulus of thin films using indentation tests, Int. J. Sol. Struct. 44 (2007) 8313-8334.

[9] H. Ogi, J. Tian, T. Tada, M. Hirao, Elastic-stiffness mapping by resonance-ultrasound microscopy with isolated piezoelectric oscillator, Appl. Phys. Lett. 83 (2003) 464-466.

[10] H. Ogi, M. Hirao, T. Tada, J. Tian, Elastic-stiffness distribution on polycrystalline copper studied by resonance ultrasound microscopy: Young's modulus microscopy, Phys. Rev. B 73 (2006) 174107. 
[11] H. Ogi, T. Inoue, H. Nagai, M. Hirao, Quantitative imaging of Young's modulus of solids: A contact-mechanics study, Rev. Sci. Instrum. 79 (2008) 053701.

[12] H. Ogi, H. Niho, M. Hirao, Internal-friction mapping on solids by resonance ultrasound microscopy, Appl. Phys. Lett. 88 (2006) 141110.

[13] H. Ogi, N. Nakamura, K. Sato, M. Hirao, S. Uda, Elastic, anelastic, and piezoelectric coefficients of langasite $\left(\mathrm{La}_{3} \mathrm{Ga}_{5} \mathrm{SiO}_{14}\right)$ : Resonance ultrasound spectroscopy with laser-Doppler interferometry, IEEE?Trans. Ultrason. Freq. Contr. 50 (2003) 553-560.

[14] J.R. Willis, Hertzian contact of anisotropic bodies, J. Mech. Phys. Solids 14 (1966) 163. 


\section{Figure Captions}

Fig. 1 (a) Rod-shape oscillator with a tip contacting specimen by the biasing force $F$, and (b) its model using the contact stiffness $K$. The contact with the specimen increases the resonance frequency of the oscillator $f$ by $\Delta f$.

Fig. 2 Relationship between the specimen Young's modulus $E_{\text {spe }}$ and the resonance frequency change when spherical tungsten-carbide tips are used. Parameters used are $E_{\text {tip }}=630 \mathrm{GPa}, \nu_{\text {tip }}=0.21, E_{\text {osc }}=118 \mathrm{GPa}$, $\nu_{\text {spe }}=0.25$ and $F=0.455 \mathrm{~N}$.

Fig. 3 (a) Laboratory-built measurement system and (b) a closeup near the tungsten-carbide tip.

Fig. 4 Appearance of the ballpoint-pen probe before (upper) and after (lower) contacting a specimen.

Fig. 5 Resonance spectra measured in contacts with various specimens.

Fig. 6 Young's moduli measured by the present method $\left(E_{\text {meas }}\right)$ and those reported $(E)$.

Fig. 7 Distribution of the strain energy caused by a contact with a (111) face of $\mathrm{Cu} . \quad a(=17.7 \mu \mathrm{m})$ denotes the contact radius. Parameters used are $E_{\text {tip }}=630 \mathrm{GPa}, \nu_{\text {tip }}=0.21, E_{\text {osc }}=118 \mathrm{GPa}, \nu_{\text {spe }}=0.25$ and $F=0.455$ N. 


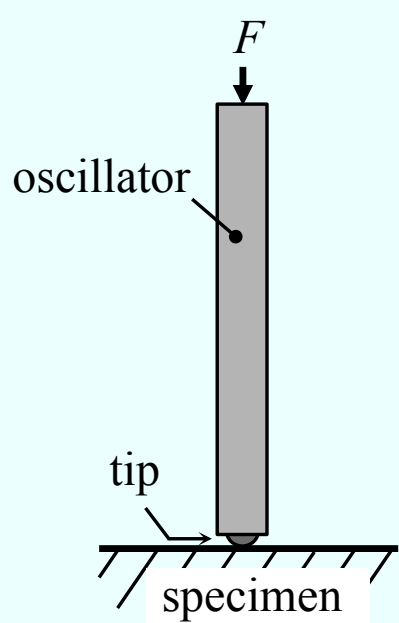

(a)

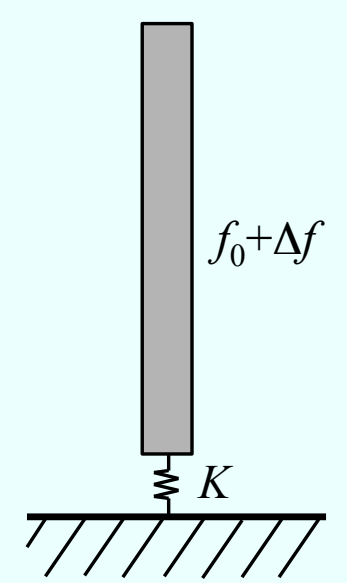

(b)

Figure 1: 


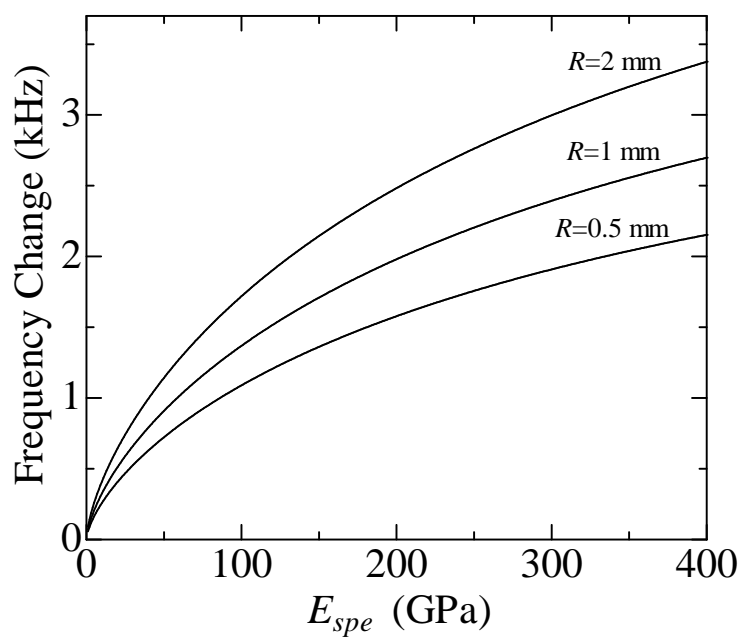

Figure 2: 

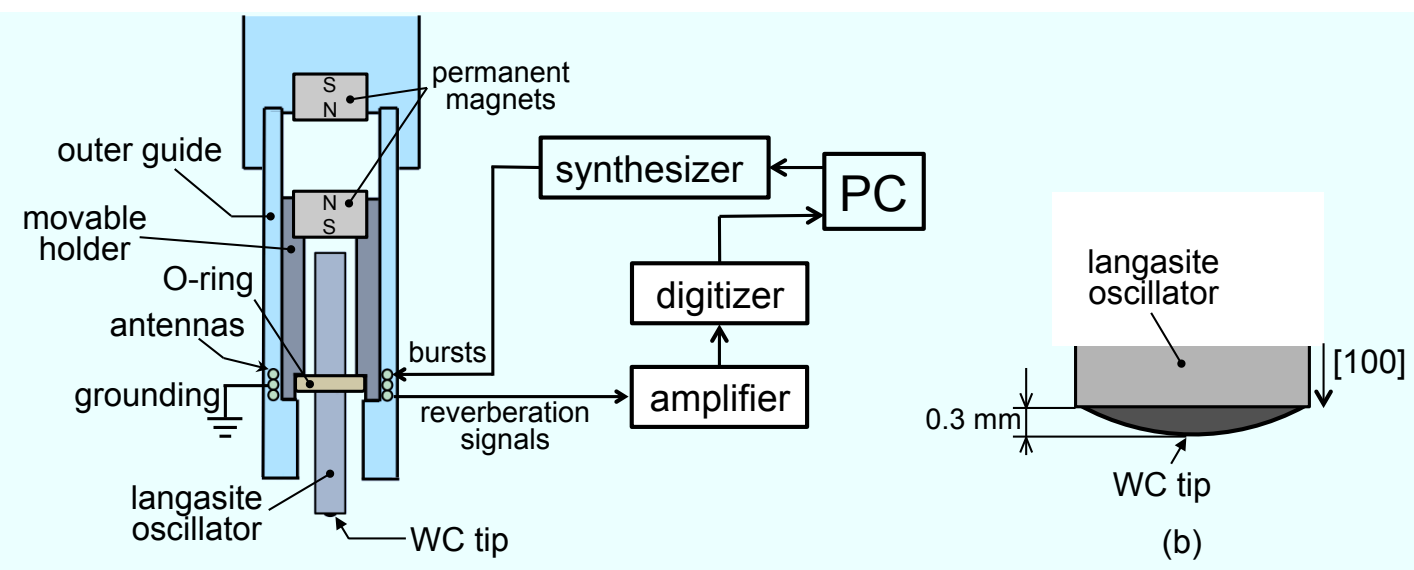

(a)

(b)

Figure 3: 


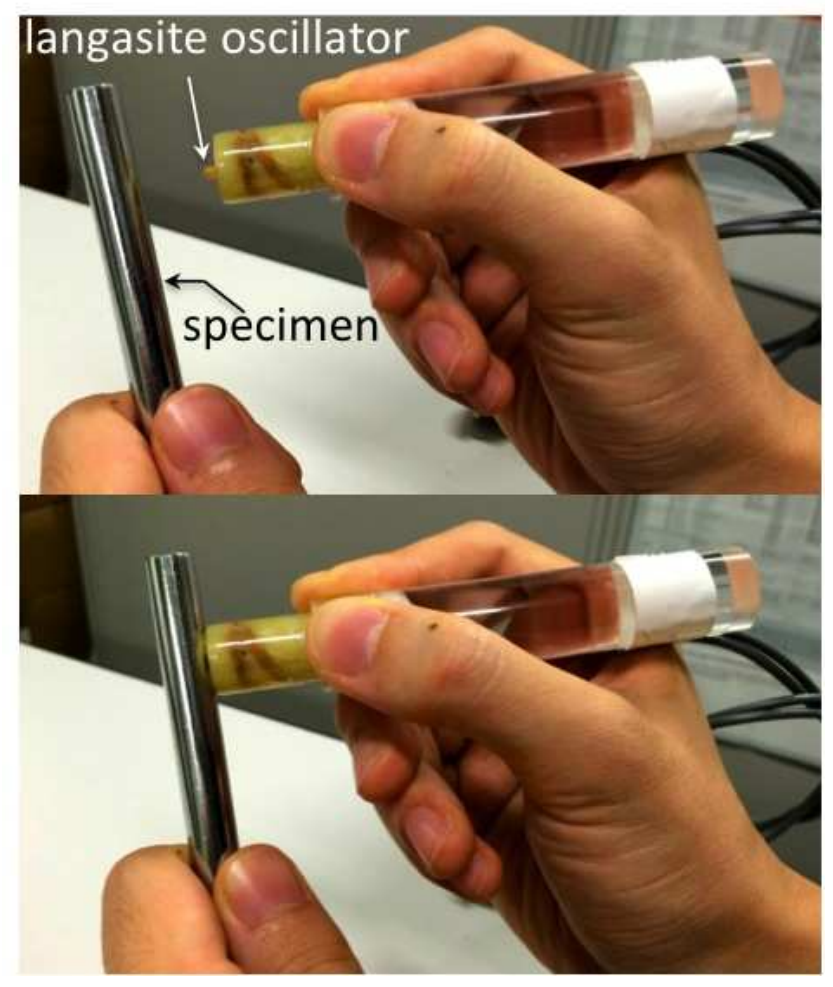

Figure 4: 


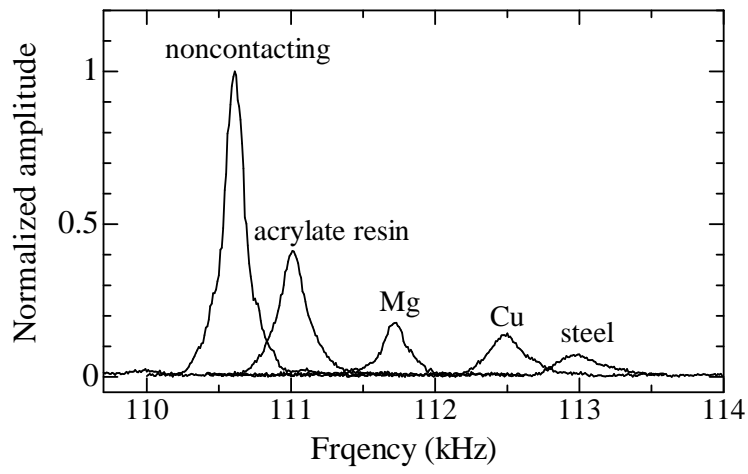

Figure 5: 


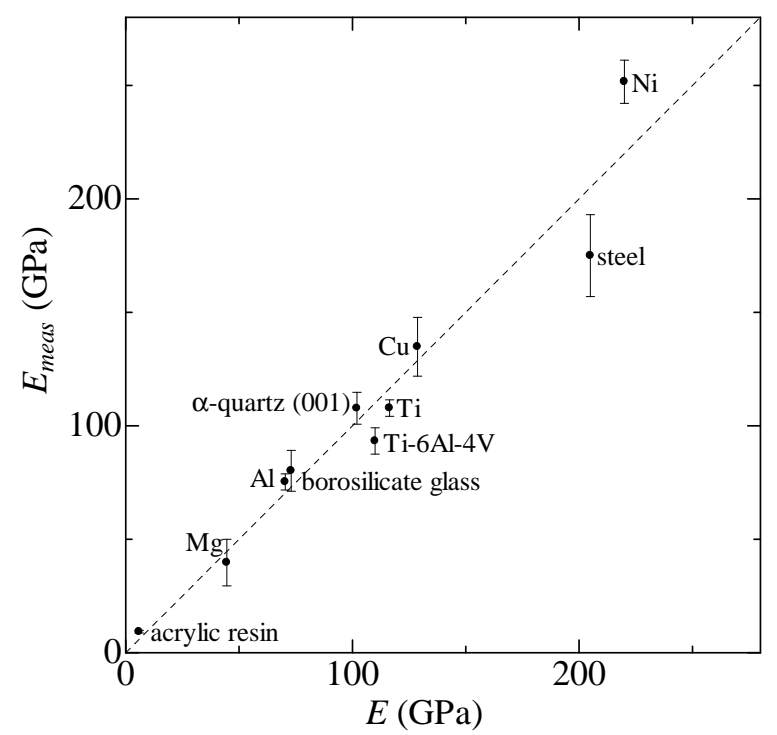

Figure 6: 


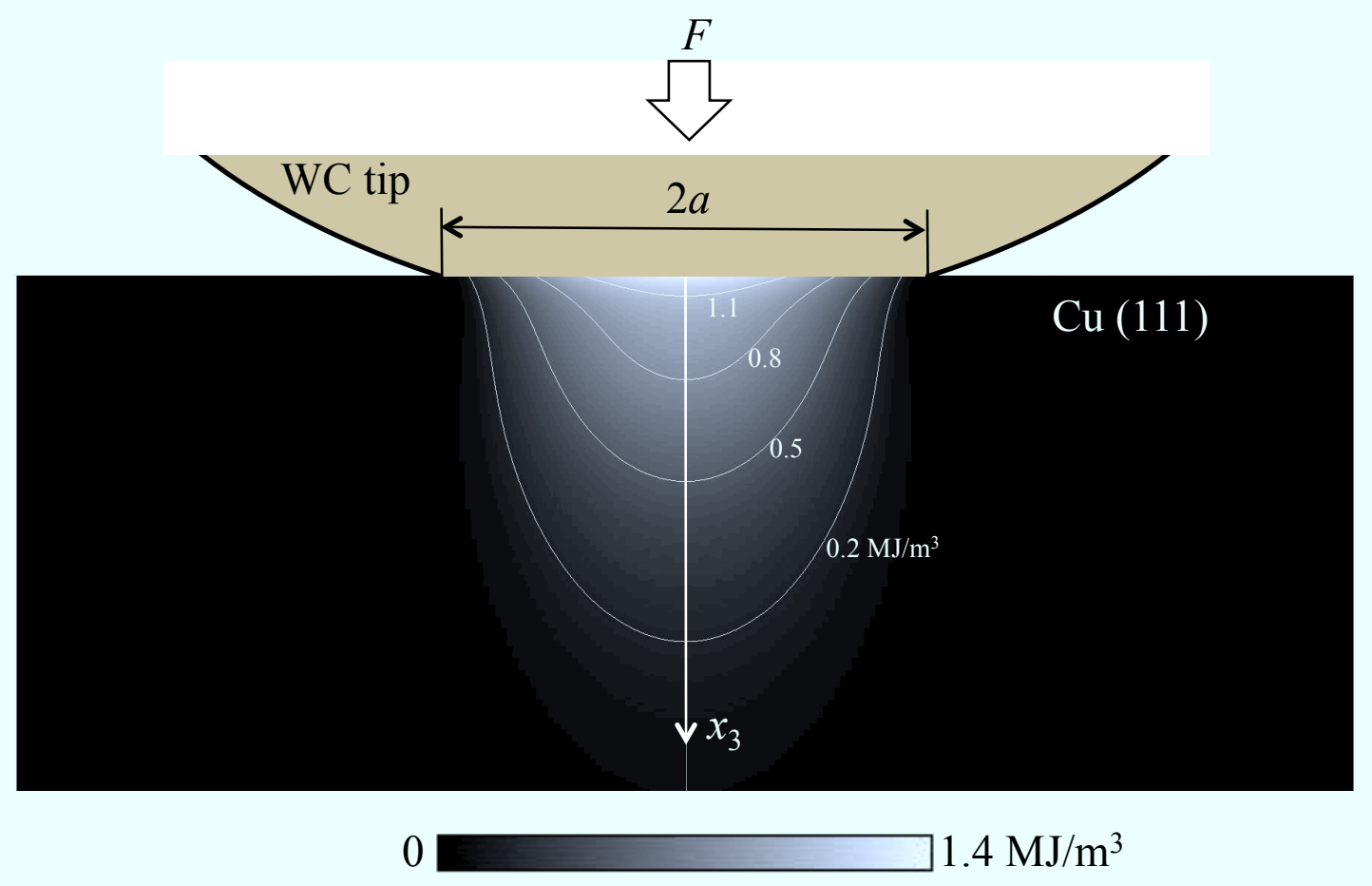

Figure 7: 
Highlights (for review)

A portable ballpoint-pen type Young's modulus tester is developed. The langasite probe is acoustically isolated.

It provides us with Young's modulus just by touching the specimen.

No parameters and reference samples are needed. 